\title{
A PRIORI ESTIMATES OF THE DEGENERATE MONGE-AMPÈRE EQUATION ON KÄHLER MANIFOLDS OF NON-NEGATIVE BISECTIONAL CURVATURE
}

\author{
SEbastien PiCARD
}

\begin{abstract}
The regularity theory of the degenerate complex Monge-Ampère equation is studied. The equation is considered on a closed compact Kähler manifold $(M, g)$ with non-negative orthogonal bisectional curvature of dimension $m$. Given a solution $\varphi$ of the degenerate complex Monge-Ampère equation $\operatorname{det}\left(g_{i \bar{j}}+\varphi_{i \bar{j}}\right)=f \operatorname{det}\left(g_{i \bar{j}}\right)$, it is shown that the Laplacian of $\varphi$ can be controlled by a constant depending on $(M, g)$, sup $f$, and $\inf _{M} \Delta f^{1 /(m-1)}$.
\end{abstract}

\section{Introduction}

We will be looking at the regularity theory of the degenerate complex Monge-Ampère equation. Let us consider the equation on a compact Kähler manifold $(M, g)$ without boundary of dimension $m$. The problem of solving the complex Monge-Ampère equation on $M$ was first motivated by the Calabi conjecture. The conjecture was reduced to solving a non-degenerate Monge-Ampère equation, and the question was solved by Yau [18]. This result had significant geometric implications, in particular, leading to the theory of Calabi-Yau manifolds, which now plays a central role in string theory and complex geometry. One of the major steps in the proof of the Calabi conjecture was establishing an a priori estimate on the Laplacian of the solution; this was done independently by Aubin [1] and Yau [18].

Although the Calabi conjecture deals with a non-degenerate Monge-Ampère equation, Yau's paper [18] also treated the degenerate Monge-Ampère equation, with an application to holomorphic sections of line bundles over $M$. More recently, the results of Yau were generalized by Kolodziej [14]. The degenerate Monge-Ampère equation in complex geometry has become an active area of research, with connections to the Minimal Model Program [5,9], or geodesics joining two Kähler potentials in the space of Kähler metrics [7,8,16]. For a survey of some of these topics, see [15].

In this paper, we shall consider the following complex Monge-Ampère equation:

$$
\operatorname{det}\left(\varphi_{i \bar{j}}+g_{i \bar{j}}\right)=f \operatorname{det} g_{i \bar{j}}
$$

where $f: M \rightarrow \mathbb{R}$ and $f \geq 0$.

The objective is the following: given a solution $\varphi$ to (1.1) such that $\left(\varphi_{i \bar{j}}+g_{i \bar{j}}\right)$ is positive semi-definite, we seek an estimate $|\Delta \varphi| \leq C$ depending only on $(M, g)$, sup $f$, and a constant $A$ such that

$$
\inf _{M} \Delta f^{\frac{1}{m-1}} \geq-A
$$

Received by the editors June 5, 2013. 
This problem is motivated by a similar result obtained for the real Monge-Ampère equation by Guan [11]. Previously, a result on Kähler manifolds was obtained by Blocki [3] while assuming that $f^{\frac{1}{m-1}} \in C^{1,1}$. Later, Blocki improved his result in [4] to requiring the assumption that $f^{\frac{1}{m-1}} \Delta(\log f)$ is bounded below, which is equivalent to

$$
f \Delta f-|\nabla f|^{2} \geq-A f^{2-\frac{1}{m-1}} .
$$

In comparison, our desired condition (1.2) is equivalent to

$$
f \Delta f-\frac{m-2}{m-1}|\nabla f|^{2} \geq-A f^{2-\frac{1}{m-1}} .
$$

We solve the problem in the case when $M$ has non-negative orthogonal bisectional curvature. Similar results were obtained by Hou [13] by obtaining a Laplacian estimate for complex Hessian equations depending on $\inf _{M} \Delta f^{\frac{1}{m}}$ in the case of non-negative orthogonal bisectional curvature. The main difficulty in improving the exponent from $1 / m$ to $1 /(m-1)$ is that we can no longer discard terms by using the concavity of $(\operatorname{det} B)^{1 / m}$, and the resulting third-order terms must be handled carefully.

We use the following definition.

Definition 1. A Kähler manifold $(M, g)$ is said to have non-negative orthogonal bisectional curvature if at each point $p \in M$, for any orthonormal basis $\left\{e_{1}, \ldots, e_{m}\right\}$ of the tangent space at $p$, we have $R\left(e_{i}, \overline{e_{i}}, e_{j}, \overline{e_{j}}\right)=R_{i \bar{i} j \bar{j}} \geq 0$.

Manifolds satisfying this curvature condition are well-understood; indeed, compact Kähler manifolds of non-negative orthogonal bisectional curvature were classified by $\mathrm{Gu}$ and Zhang [10]. Our main theorem is the following.

Theorem 2. Let $(M, g)$ be a compact Kähler manifold with non-negative orthogonal bisectional curvature and empty boundary. Let $f>0$ be a function on $M$ such that $\inf _{M} \Delta f^{\frac{1}{m-1}} \geq-A$ for some constant $A$. For all solutions $\varphi \in C^{4}(M)$ of

$$
\operatorname{det}\left(g_{i \bar{j}}+\varphi_{i \bar{j}}\right)=f \operatorname{det} g_{i \bar{j}},
$$

such that $\left(\varphi_{i \bar{j}}+g_{i \bar{j}}\right)$ is positive definite, we have

$$
\left(\sup _{M} \varphi-\inf _{M} \varphi\right)+\|\nabla \varphi\|_{\infty}+\|\Delta \varphi\|_{\infty} \leq C,
$$

where $C$ depends on $(M, g), A$, and $\sup f$.

Although this theorem assumes $f>0$, such an estimate is applicable to the degenerate case when $f \geq 0$ via a limiting process. This shall be illustrated in Section 3 , which contains a proof of the following application.

Using the a priori estimates, we solve the Dirichlet problem for the degenerate complex Monge-Ampère equation on a domain $\Omega$ in $\mathbb{C}^{m}$. This type of question was investigated by Bedford and Taylor [2], and Caffarelli, Kohn, Niremberg and Spruck [6]. The problem is of the following form:

$$
\begin{gathered}
\operatorname{det} u_{i \bar{j}}(z)=f(z) \text { in } \Omega, \\
u=0 \text { on } \partial \Omega .
\end{gathered}
$$

Before stating our result, we first establish some terminology. We say a real-valued function $u$ is pluri-subharmonic if $\left(u_{i \bar{j}}\right)$ is positive semi-definite. We say a real-valued function $u$ is strictly pluri-subharmonic if $\left(u_{i \bar{j}}\right)$ is positive definite. Following the 
terminology of Blocki [3], if $|\Delta u|$ is bounded, we say $u$ is almost $C^{1,1}$. A domain $\Omega \subset \mathbb{C}^{m}$ with smooth boundary $\partial \Omega$ is called strongly pseudo-convex if there exists a smooth real-valued function $r$ defined on a neighbourhood of $\bar{\Omega}$ such that $r<0$ in $\Omega$, $r=0$ on $\partial \Omega, r>0$ outside of $\bar{\Omega}, d r \neq 0$, and $\left(r_{i \bar{j}}(z)\right)$ is positive-definite at each point in its domain. With these definitions in place, we can now state the following result.

Theorem 3. Let $\Omega$ be a bounded strongly pseudo-convex domain in $\mathbb{C}^{m}$. Let $f: \Omega \rightarrow$ $\mathbb{R}$ be a function such that $f \geq 0,\left|\nabla f^{1 / m}\right| \leq A_{1}$, and $\Delta f^{\frac{1}{m-1}} \geq-A_{2}$. Then there exists a unique pluri-subharmonic, almost $C^{1,1}$ solution $u$ such that

$$
\begin{aligned}
& \operatorname{det} u_{i \bar{j}}(z)=f(z) \text { in } \Omega, \\
& u=0 \text { on } \partial \Omega .
\end{aligned}
$$

Furthermore, $\|u\|_{C^{1}(\bar{\Omega})}+\|\Delta u\|_{\infty} \leq C$, where $C$ depends only on $A_{1}, A_{2}, \sup (f)$, and $\Omega$.

\section{Preliminaries}

In this section, we establish some notation and recall previous results relating to the proof of Theorem 2. First, we remind the reader that the constant $C$ denotes a positive quantity that is under control, and may change line by line. We will use the convention $\varphi_{i \bar{j}}=\frac{\partial^{2} \varphi}{\partial z^{i} \partial \bar{z}^{j}}$; as opposed to Yau [18], subscripts do not indicate covariant derivatives. Also, for a function $h: M \rightarrow \mathbb{C}$, we will use any of the following notation interchangeably: $\frac{\partial h}{\partial z^{i}}, \partial_{i} h$, and $h_{i}$. We shall denote

$$
g_{i \bar{j}}^{\prime}:=g_{i \bar{j}}+\varphi_{i \bar{j}}
$$

It is well-known, as seen, for example, in the exposition of Siu [17], that for Theorem 2 we have an a priori estimate

$$
\left(\sup _{M} \varphi-\inf _{M} \varphi\right) \leq C
$$

The objective of this paper is to estimate $|\Delta \varphi| \leq C$ directly from the uniform bound. Assuming such a bound on the Laplacian, we can obtain a bound on the gradient. Indeed, if we look at $\Delta \varphi(z):=G(z)$, then $|G(z)| \leq C$. Then by the Schauder estimates

$$
\sup _{M}|\nabla \varphi| \leq C_{0}\left(\|G\|_{\infty}+\|\varphi\|_{2}\right) \leq C
$$

Furthermore, we can easily obtain a lower bound on $\Delta \varphi$. Since $g_{i \bar{j}}^{\prime}=g_{i \bar{j}}+\varphi_{i \bar{j}}$ is positive definite, we have $0<\operatorname{Tr}\left(g_{i \bar{j}}+\varphi_{i \bar{j}}\right)$. At any point $p \in M$, we may choose coordinates such that $g_{i \bar{j}}=\delta_{i \bar{j}}$. Thus

$$
0<m+\Delta \varphi,
$$

and it only remains to bound $\Delta \varphi$ from above. 


\section{Second-order estimate}

As shown in the previous section, Theorem 2 will following from the following estimate.

Proposition 4. Let $(M, g)$ be a closed, compact Kähler manifold with non-negative orthogonal bisectional curvature. Let $f>0$ be a positive function on $M$ such that $\inf _{M} \Delta f^{\frac{1}{m-1}} \geq-A$ for some constant A. For all $\varphi \in C^{4}(M)$ satisfying (1.1) such that $\left(\varphi_{i \bar{j}}+g_{i \bar{j}}\right)$ is positive-definite, we have

$$
|\Delta \varphi| \leq C
$$

where $C$ depends on $(M, g),(\sup \varphi-\inf \varphi), A$, and $\sup f$.

Proof. We will estimate the maximum value of the following test function:

$$
H=(m+\Delta \varphi) e^{-\alpha(\varphi)},
$$

where $\alpha:[2, \lambda] \rightarrow \mathbb{R}$ is a function that will be specified later. In view of the $L^{\infty}$ estimate, we may shift $\varphi$ by a constant and assume that $\varphi(p) \in[2, \lambda]$ for all $p \in M$. We start by computing the first two derivatives of $H$.

$$
\begin{aligned}
H_{\gamma}= & (\Delta \varphi)_{\gamma} e^{-\alpha(\varphi)}-\alpha^{\prime}(m+\Delta \varphi) \varphi_{\gamma} e^{-\alpha(\varphi)}, \\
H_{\gamma \bar{\gamma}}= & \left((\Delta \varphi)_{\gamma \bar{\gamma}}-\alpha^{\prime}(m+\Delta \varphi) \varphi_{\gamma \bar{\gamma}}-\alpha^{\prime \prime}(m+\Delta \varphi) \varphi_{\gamma} \varphi_{\bar{\gamma}}\right) e^{-\alpha(\varphi)} \\
& +\left(-\alpha^{\prime}\left((\Delta \varphi)_{\gamma} \varphi_{\bar{\gamma}}+\overline{(\Delta \varphi)_{\gamma}} \varphi_{\gamma}\right)+\left(\alpha^{\prime}\right)^{2}(m+\Delta \varphi) \varphi_{\gamma} \varphi_{\bar{\gamma}}\right) e^{-\alpha(\varphi)} .
\end{aligned}
$$

Let $p \in M$ be the point where $H$ achieves its maximum value. Since the manifold is Kähler, we may choose coordinates such that at $p$ we have $g_{i \bar{j}}=\delta_{i j}, \frac{\partial}{\partial z^{k}} g_{i \bar{j}}=0$ and $\varphi_{i \bar{j}}=\delta_{i j} \varphi_{i \bar{j}}$. At $p$, the gradient of $H$ is equal to zero, and hence,

$$
(\Delta \varphi)_{\gamma}=\alpha^{\prime} \varphi_{\gamma}(m+\Delta \varphi)
$$

We recall the notation (2.1). Since $g^{\prime}$ defines a Kähler metric on $M$, we denote $\Delta^{\prime}=g^{\prime i \bar{j}} \partial_{i} \bar{\partial}_{j}$ to be the Laplacian of $\left(M, g^{\prime}\right)$. By the maximum principle, $\Delta^{\prime} H(p) \leq 0$, hence if we use this fact while substituting the gradient equation (3.3) into (3.2), we obtain

$$
\begin{aligned}
\Delta^{\prime} \Delta \varphi \leq & \alpha^{\prime}(m+\Delta \varphi) \Delta^{\prime} \varphi+\left(\alpha^{\prime}\right)^{2}(m+\Delta \varphi) g^{\prime i \bar{i}} \varphi_{i} \varphi_{\bar{i}} \\
& +\alpha^{\prime \prime}(m+\Delta \varphi) g^{\prime i \bar{i}} \varphi_{i} \varphi_{\bar{i}} .
\end{aligned}
$$

Next, we raise both sides of $(1.1)$ to the power of $1 /(m-1)$ and take derivatives. This yields

$$
\begin{aligned}
& (m-1)\left(\operatorname{det} g_{i \bar{j}}\right)^{\frac{1}{m-1}} \partial_{\gamma} f^{\frac{1}{m-1}}+f^{\frac{1}{m-1}}\left(\operatorname{det} g_{i \bar{j}}\right)^{\frac{1}{m-1}} g^{i \bar{j}} \partial_{\gamma} g_{i \bar{j}} \\
& \quad=\left(\operatorname{det} g_{i \bar{j}}^{\prime}\right)^{\frac{1}{m-1}} g^{\prime i \bar{j}}\left(\partial_{\gamma} g_{i \bar{j}}+\varphi_{i \bar{j} \gamma}\right) .
\end{aligned}
$$

We then take another derivative of the previous equation, noting that our choice of coordinates will simplify the expression.

$$
\begin{aligned}
& (m-1) \partial_{\gamma} \bar{\partial}_{\gamma} f^{\frac{1}{m-1}}+f^{\frac{1}{m-1}} \delta^{i \bar{j}} \partial_{\gamma} \bar{\partial}_{\gamma} g_{i \bar{j}}=\frac{1}{m-1} f^{\frac{1}{m-1}} g^{\prime i \bar{i}} g^{\prime j \bar{j}} \varphi_{i \bar{i} \gamma} \varphi_{j \bar{j} \bar{\gamma}} \\
& \quad+f^{\frac{1}{m-1}}\left(g^{\prime i \bar{i}}\left(\partial_{\gamma} \bar{\partial}_{\gamma} g_{i \bar{i}}+\varphi_{i \bar{i} \gamma \bar{\gamma}}\right)+\varphi_{i \bar{j} \gamma} \bar{\partial}_{\gamma} g^{\prime i \bar{j}}\right)
\end{aligned}
$$


Expanding out $\partial_{\gamma} g^{\prime i \bar{j}}$ and using the definition of the curvature tensor, we obtain

$$
\begin{aligned}
& (m-1) f^{\frac{-1}{m-1}} \partial_{\gamma} \bar{\partial}_{\gamma} f^{\frac{1}{m-1}}-\delta^{i \bar{j}} R_{i \bar{j} \gamma \bar{\gamma}} \\
& \quad=\frac{1}{m-1} g^{\prime i \bar{i}} g^{\prime j \bar{j}} \varphi_{i \bar{\gamma} \gamma} \varphi_{j \bar{j} \bar{\gamma}}-g^{\prime i \bar{i}} R_{i \bar{i} \gamma \bar{\gamma}}+g^{\prime i \bar{i}} \varphi_{i \bar{i} \gamma \bar{\gamma}}-g^{\prime i \bar{i}} g^{\prime j \bar{j}} \varphi_{i \bar{j} \gamma} \varphi_{j \bar{i} \bar{\gamma}}
\end{aligned}
$$

Also, at the point in consideration we have

$$
\begin{aligned}
\Delta^{\prime} \Delta \varphi & =g^{\prime k \bar{l}} \partial_{k} \bar{\partial}_{l}\left(g^{i \bar{j}} \varphi_{i \bar{j}}\right) \\
& =g^{\prime k \bar{l}} \varphi_{i \bar{j}} \partial_{k} \bar{\partial}_{l} g^{i \bar{j}}+g^{\prime k \bar{l}} g^{i \bar{j}} \varphi_{i \bar{j} k \bar{l}} \\
& =-g^{\prime k \bar{l}} g^{i \bar{t}} g^{n \bar{j}} \varphi_{i \bar{j}} \partial_{k} \bar{\partial}_{l} g_{n \bar{t}}+g^{\prime k \bar{l}} g^{i \bar{j}} \varphi_{i \bar{j} k \bar{l}} \\
& =\sum_{k, i} g^{\prime k \bar{k}} \varphi_{i \bar{i}} R_{i \bar{i} k \bar{k}}+\sum_{k, i} g^{\prime i \bar{i}} \varphi_{i \bar{i} k \bar{k}}
\end{aligned}
$$

After summing the $\gamma$ in (3.5) and substituting the previous identity, one obtains the following at the point $p$ :

$$
\begin{aligned}
(m-1) f^{\frac{-1}{m-1}} \Delta f^{\frac{1}{m-1}}= & \Delta^{\prime} \Delta \varphi+\sum_{k} \frac{1}{m-1} g^{\prime i \bar{i}} g^{\prime j \bar{j}} \varphi_{i \bar{i} k} \varphi_{j \bar{j} \bar{k}}-\sum_{k} g^{\prime i \bar{i}} g^{\prime j \bar{j}} \varphi_{i \bar{j} k} \varphi_{i \bar{j} \bar{k}} \\
& -\sum_{k} g^{\prime i \bar{i}}\left(1+\varphi_{k \bar{k}}\right) R_{i \bar{i} k \bar{k}}+\sum_{i, k} R_{i \bar{i} k \bar{k}}
\end{aligned}
$$

We substitute (3.4), define $S:=\sum_{i, k} R_{i \bar{i} k \bar{k}}$, and obtain

$$
\begin{aligned}
(m-1) f^{\frac{-1}{m-1}} \Delta f^{\frac{1}{m-1}} \leq & \alpha^{\prime} m(m+\Delta \varphi)-\alpha^{\prime}(m+\Delta \varphi)\left(\sum_{i} g^{\prime i \bar{i}}\right) \\
& +\left(\alpha^{\prime}\right)^{2}(m+\Delta \varphi) g^{\prime i \bar{i}} \varphi_{i} \varphi_{\bar{i}}+\alpha^{\prime \prime}(m+\Delta \varphi) g^{\prime i \bar{i}} \varphi_{i} \varphi_{\bar{i}} \\
& +\sum_{k} \frac{1}{m-1} g^{\prime i \bar{i}} g^{\prime j \bar{j}} \varphi_{i \bar{i} k} \varphi_{j \bar{j} \bar{k}}-\sum_{k} g^{\prime i \bar{i}} g^{\prime j \bar{j}} \varphi_{\bar{i} j k} \varphi_{i \bar{j} \bar{k}} \\
& -\inf _{i, k} R_{i \bar{i} k \bar{k}}(m+\Delta \varphi)\left(\sum_{i} g^{\prime i \bar{i}}\right)+S .
\end{aligned}
$$

If $M$ has non-negative orthogonal bisectional curvature, then $\inf _{i, k} R_{i \bar{i} k \bar{k}}$ is nonnegative and the term involving it can be dropped. We are left with

$$
\begin{aligned}
(m-1) f^{\frac{-1}{m-1}} \Delta f^{\frac{1}{m-1}} \leq & \alpha^{\prime} m(m+\Delta \varphi)+S-\alpha^{\prime}(m+\Delta \varphi)\left(\sum_{i} g^{\prime i \bar{i}}\right) \\
& +\left(\alpha^{\prime \prime}+\left(\alpha^{\prime}\right)^{2}\right)(m+\Delta \varphi) g^{\prime i \bar{i}} \varphi_{i} \varphi_{\bar{i}} \\
& +\sum_{k} \frac{1}{m-1} g^{\prime i \bar{i}} g^{\prime j \bar{j}} \varphi_{i \bar{i} k} \varphi_{j \bar{j} \bar{k}}-\sum_{k} g^{\prime i \bar{i}} g^{\prime j \bar{j}} \varphi_{i \bar{j} k} \varphi_{i \bar{j} \bar{k}} .
\end{aligned}
$$

The troublesome terms are those involving third-order derivatives, and we shall follow the argument of Guan [11] to control the following quantity for a fixed $k$ :

$$
\frac{1}{m-1} g^{\prime i \bar{i}} g^{\prime j \bar{j}} \varphi_{i \bar{i} k} \varphi_{j \bar{j} \bar{k}}-g^{\prime i \bar{i}} g^{\prime j \bar{j}} \varphi_{i \bar{j} j k} \varphi_{i \bar{j} \bar{k}}
$$


First, we drop mixed terms $\left|\varphi_{\bar{i} j k}\right|^{2}$ for $i \neq j$ and obtain

$$
\frac{1}{m-1} g^{\prime i \bar{i}} g^{\prime j \bar{j}} \varphi_{i \bar{i} k} \varphi_{j \bar{j} \bar{k}}-g^{\prime i \bar{i}} g^{\prime j \bar{j}} \varphi_{\bar{i} j k} \varphi_{i \bar{j} \bar{k}} \leq \frac{1}{m-1}\left|g^{\prime i \bar{i}} \varphi_{i \bar{i} k}\right|^{2}-\left(g^{\prime i \bar{i}}\right)^{2}\left|\varphi_{i \bar{i} k}\right|^{2}
$$

We recall that $\varphi_{i \bar{i}}(z)$ is a locally defined real-valued function. Also, $\varphi_{i \bar{i} k}=\partial_{z^{k}} \varphi_{i \bar{i}}$ where $\partial_{z^{k}}=\frac{1}{2}\left(\partial_{x^{k}}-i \partial_{y^{k}}\right)$. Thus

$$
\left|\varphi_{i \bar{i} k}\right|^{2}=\frac{1}{4}\left(\varphi_{i \bar{i} x}^{2}+\varphi_{i \bar{i} y}^{2}\right)
$$

where we write $f_{x}$ for $\partial_{x^{k}} f$, and there is no confusion since $k$ is fixed. Thus we get

$$
\begin{aligned}
& \frac{4}{m-1} g^{\prime i \bar{i}} g^{\prime j \bar{j}} \varphi_{i \bar{i} k} \varphi_{j \bar{j} \bar{k}}-4 g^{\prime i \bar{i}} g^{\prime j \bar{j}} \varphi_{i \bar{j} k} \varphi_{i \bar{j} \bar{k}} \\
& \leq \frac{1}{m-1}\left(\sum_{i} g^{\prime i \bar{i}} \varphi_{i \bar{i} x}\right)^{2}-\sum_{i}\left(g^{\prime i \bar{i}} \varphi_{i \bar{i} x}\right)^{2} \\
& \quad+\frac{1}{m-1}\left(\sum_{i} g^{\prime i \bar{i}} \varphi_{i \bar{i} y}\right)^{2}-\sum_{i}\left(g^{\prime i \bar{i}} \varphi_{i \bar{i} y}\right)^{2}
\end{aligned}
$$

We shall show how to control the terms containing real derivatives in the $x$ direction. Let $I=\left\{1 \leq i \leq m: \varphi_{i \bar{i} x}(p)>0\right\}$ and $J=\left\{1 \leq i \leq m: \varphi_{i \bar{i} x}(p)<0\right\}$. We consider the two following cases. Case 1: $I$ and $J$ are both non-empty, or case 2: either $I$ or $J$ is empty. In case 1 , we have $|I| \leq m-1$ and $|J| \leq m-1$. Using $\left(\sum_{i}^{n} a_{i}\right)^{2} \leq n \sum a_{i}^{2}$ for $a_{i} \geq 0$, we can compute the following:

$$
\begin{aligned}
& \frac{1}{m-1}\left(\sum_{i} g^{\prime i \bar{i}} \varphi_{i \bar{i} x}\right)^{2}-\sum_{i}\left(g^{\prime i \bar{i}} \varphi_{i \bar{i} x}\right)^{2} \\
& \quad=\frac{1}{m-1}\left(\left(\sum_{I} g^{\prime i \bar{i}} \varphi_{i \bar{i} x}\right)^{2}+\left(\sum_{J} g^{\prime i \bar{i}} \varphi_{i \bar{i} x}\right)^{2}+2\left(\sum_{I} g^{\prime i \bar{i}} \varphi_{i \bar{i} x}\right)\left(\sum_{J} g^{\prime i \bar{i}} \varphi_{i \bar{i} x}\right)\right) \\
& \quad-\sum_{I}\left(g^{\prime i \bar{i}} \varphi_{i \bar{i} x}\right)^{2}-\sum_{J}\left(g^{\prime i \bar{i}} \varphi_{i \bar{i} x}\right)^{2} \\
& \leq \frac{1}{m-1}\left(\sum_{I} g^{\prime i \bar{i}} \varphi_{i \bar{i} x}\right)^{2}-\sum_{I}\left(g^{\prime \prime \bar{i}} \varphi_{i \bar{i} x}\right)^{2}+\frac{1}{m-1}\left(\sum_{J} g^{\prime i \bar{i}} \varphi_{i \bar{i} x}\right)^{2}-\sum_{J}\left(g^{\prime i \bar{i}} \varphi_{i \bar{i} x}\right)^{2} \\
& \leq 0 .
\end{aligned}
$$

Case 2 is a little bit more delicate. Without loss of generality, we assume that $J=\emptyset$. Therefore, $\varphi_{i \bar{i} x}(p)>0$ for all $i$. Using (3.3), we obtain the following at $p$ :

$$
\varphi_{i \bar{i} x} \leq \sum_{j=1}^{m} \varphi_{j \bar{j} x}=2 R e\left(\frac{\partial}{\partial z^{k}} \Delta \varphi\right) \leq 2\left|(\Delta \varphi)_{k}\right| \leq 2 \alpha^{\prime}|\nabla \varphi|(m+\Delta \varphi) .
$$


We now compute

$$
\begin{aligned}
& \frac{1}{m-1}\left(\sum_{i} g^{\prime i \bar{i}} \varphi_{i \bar{i} x}\right)^{2}-\sum_{i}\left(g^{\prime i \bar{i}} \varphi_{i \bar{i} x}\right)^{2} \\
& =\frac{1}{m-1}\left(\sum_{i=1}^{m-1} g^{\prime i \bar{i}} \varphi_{i \bar{i} x}+g^{\prime m \bar{m}} \varphi_{m \bar{m} x}\right)^{2}-\sum_{i=1}^{m}\left(g^{\prime i \bar{i}} \varphi_{i \bar{i} x}\right)^{2} \\
& =\frac{2}{m-1} g^{\prime m \bar{m}} \varphi_{m \bar{m} x} \sum_{i=1}^{m-1} g^{\prime i \bar{i}} \varphi_{i \bar{i} x}+\frac{1}{m-1}\left(\sum_{i=1}^{m-1} g^{\prime i \bar{i}} \varphi_{i \bar{i} x}\right)^{2}-\sum_{i=1}^{m-1}\left(g^{\prime i \bar{i}} \varphi_{i \bar{i} x}\right)^{2} \\
& \quad+\frac{1}{m-1}\left(g^{\prime m \bar{m}} \varphi_{m \bar{m} x}\right)^{2}-\left(g^{\prime m \bar{m}} \varphi_{m \bar{m} x}\right)^{2}
\end{aligned}
$$

Without loss of generality, we can assume $\varphi_{m \bar{m}}(p) \geq \varphi_{i \bar{i}}(p)$ for all $i$. Therefore, using (3.10) we have

$$
\begin{aligned}
\frac{1}{m-1}\left(\sum_{i} g^{\prime i \bar{i}} \varphi_{i \bar{i} x}\right)^{2}-\sum_{i}\left(g^{\prime i \bar{i}} \varphi_{i \bar{i} x}\right)^{2} & \leq \frac{2}{m-1} g^{\prime m \bar{m}} \varphi_{m \bar{m} x} \sum_{i=1}^{m-1} g^{\prime i \bar{i}} \varphi_{i \bar{i} x} \\
& \leq \frac{8}{m-1}\left(\alpha^{\prime}\right)^{2}|\nabla \varphi|^{2}(m+\Delta \varphi)^{2} g^{\prime m \bar{m}} \sum_{i=1}^{m-1} g^{\prime i \bar{i}} \\
& \leq \frac{8 m}{m-1}\left(\alpha^{\prime}\right)^{2}|\nabla \varphi|^{2}(m+\Delta \varphi) \sum_{i=1}^{m} g^{\prime i \bar{i}}
\end{aligned}
$$

The terms involving $y$ derivatives in (3.9) can be controlled in the same way as the $x$ derivatives. Thus combining both cases and (3.9), we obtain

$$
\begin{gathered}
\frac{1}{m-1} g^{\prime i \bar{i}} g^{\prime j \bar{j}} \varphi_{i \bar{i} k} \varphi_{j \bar{j} \bar{k}}-g^{\prime i \bar{i}} g^{\prime j \bar{j}} \varphi_{\bar{i} j k} \varphi_{i \bar{j} \bar{k}} \\
\leq \frac{4 m}{m-1}\left(\alpha^{\prime}\right)^{2}|\nabla \varphi|^{2}(m+\Delta \varphi) \sum_{i=1}^{m} g^{\prime i \bar{i}} .
\end{gathered}
$$

We substitute (3.11) into (3.7) and obtain

$$
\begin{aligned}
(m-1) f^{\frac{-1}{m-1}} \Delta f^{\frac{1}{m-1}} \leq & \alpha^{\prime} m(m+\Delta \varphi)+S-\alpha^{\prime}(m+\Delta \varphi)\left(\sum_{i} g^{\prime i \bar{i}}\right) \\
& +\left(\alpha^{\prime \prime}+\left(\alpha^{\prime}\right)^{2}\left(1+\frac{4 m^{2}}{m-1}\right)\right)(m+\Delta \varphi)|\nabla \varphi|^{2}\left(\sum_{i} g^{\prime i \bar{i}}\right) .
\end{aligned}
$$

Denote $C_{0}:=1+4 m^{2} /(m-1)$. Following an idea of Blocki in his gradient estimate [4], we pick $\alpha(x)=\left(C_{0}\right)^{-1} \log x$. We know that $\alpha(\varphi)$ is well-defined, since $\varphi$ was renormalized such that $2 \leq \varphi \leq \lambda$. This choice of $\alpha$ yields $\alpha^{\prime \prime}+C_{0}\left(\alpha^{\prime}\right)^{2}=0$ and hence we are left with

$$
(m-1) f^{\frac{-1}{m-1}} \Delta f^{\frac{1}{m-1}} \leq \frac{1}{2 C_{0}} m(m+\Delta \varphi)+S-\frac{1}{C_{0} \lambda}(m+\Delta \varphi)\left(\sum_{i} g^{\prime i \bar{i}}\right)
$$


We next notice that for $B_{i}>0$, the following inequality holds:

$$
\left(\sum_{i=1}^{m} \frac{1}{B_{i}}\right)^{m-1} \geq \frac{\sum_{i=1}^{m} B_{i}}{\prod_{i=1}^{m} B_{i}}
$$

Since $g^{i \bar{i}}>0$, we thus have at the point $p$,

$$
\sum_{i} g^{\prime i \bar{i}} \geq\left(\frac{m+\Delta \varphi}{f}\right)^{\frac{1}{m-1}} .
$$

Substituting (3.14) into (3.13) and using the definition of $A$, we get

$$
\begin{aligned}
A(m-1) \geq & \frac{1}{C_{0} \lambda}(m+\Delta \varphi)^{1+\frac{1}{m+1}}-S \sup _{M} f^{\frac{1}{m+1}} \\
& -\left(\frac{m}{2 C_{0}} \sup _{M} f^{\frac{1}{m+1}}\right)(m+\Delta \varphi) .
\end{aligned}
$$

Thus there are constants $C_{1}, C_{2}$ under control such that

$$
(m+\Delta \varphi(p))^{1+1 /(m-1)} \leq C_{1}(m+\Delta \varphi(p))+C_{2} .
$$

It follows that there exists a constant $C_{3}$ under control such that

$$
m+\Delta \varphi(p) \leq C_{3} \text {. }
$$

Now that we have control of $(m+\Delta \varphi)$ at $p$, we have control of $(m+\Delta \varphi)$ at all $z \in M$. Indeed,

$$
(m+\Delta \varphi(z)) e^{-\alpha(\varphi(z))} \leq(m+\Delta \varphi(p)) e^{-\alpha(\varphi(p))} \leq C_{3} e^{-\alpha(\varphi(p))} .
$$

Since $\alpha(x)=C_{0}^{-1} \log x$, we have

$$
(m+\Delta \varphi(z)) \leq C_{3}\left(\frac{\lambda}{2}\right)^{1 / C_{0}}
$$

By dropping the assumption on the bisectional curvature of $M$, the curvature terms break down the previous argument. It is unknown whether Proposition 4 holds without this condition on the curvature. Before ending this section, we give a partial result working towards the removal of this assumption. To attempt to control these curvature terms, we strengthen our hypothesis to match the direct gradient estimate given by Blocki [4] or Guan [12]: we assume $f^{1 / m}$ is Lipschitz continuous. In the case $m=2$, this additional assumption makes dealing with the terms (3.8) particularly easy, and we can thus obtain the following estimate.

Proposition 5. Let $(M, g)$ be a closed, compact Kähler manifold of dimension $m=2$. Let $f>0$ be a positive function on $M$ such that $\inf _{M} \Delta f \geq-A$ for some constant $A$, and $f^{\frac{1}{2}}$ is Lipschitz. For all $\varphi \in C^{4}(M)$ satisfying (1.1) such that $\left(\varphi_{i \bar{j}}+g_{i \bar{j}}\right)$ is positive-definite, we have

$$
|\Delta \varphi| \leq C
$$

where $C$ depends on $(M, g),(\sup \varphi-\inf \varphi), A$, the Lipschitz constant of $f^{\frac{1}{2}}$, and $\sup f$. 
Proof. We run the same argument as the proof of Proposition 4 up until equation (3.6). In this case, we can simply let $\alpha(x)=\alpha_{0} x$, where $0<\alpha_{0}$ is a constant. Equation (3.6) becomes

$$
\begin{aligned}
f^{\frac{-1}{m-1}} \Delta\left(f^{\frac{1}{m-1}}\right) \leq & \alpha_{0} m(m+\Delta \varphi)+S-\left(\alpha_{0}+\inf _{i, k} R_{i \bar{i} k \bar{k}}\right)(m+\Delta \varphi)\left(\sum_{i} g^{\prime i \bar{i}}\right) \\
& +\left(\alpha_{0}\right)^{2}(m+\Delta \varphi) g^{\prime i \bar{i}} \varphi_{i} \varphi_{\bar{i}}+\sum_{k} \frac{1}{m-1} g^{\prime i \bar{i}} g^{\prime j \bar{j}} \varphi_{i \bar{i} k} \varphi_{j \bar{j} \bar{k}} \\
& -\sum_{k} g^{\prime i \bar{i}} g^{\prime j \bar{j}} \varphi_{\bar{i} j k} \varphi_{i \bar{j} \bar{k}} .
\end{aligned}
$$

We see that if we choose $\alpha_{0}>\inf _{i, k} R_{i \bar{i} k \bar{k}}$, the coefficient on the third term is negative. To eliminate the $\alpha_{0}^{2}$ term, we substitute the gradient equation (3.3):

$$
\begin{aligned}
f^{\frac{-1}{m-1}} \Delta\left(f^{\frac{1}{m-1}}\right) \leq & \alpha_{0} m(m+\Delta \varphi)+S-\left(\alpha_{0}+\inf _{i, k} R_{i \bar{i} k \bar{k}}\right)(m+\Delta \varphi)\left(\sum_{i} g^{\prime i \bar{i}}\right) \\
& +(m+\Delta \varphi)^{-1} g^{\prime i \bar{i}}(\Delta \varphi)_{i}(\Delta \varphi)_{\bar{i}}+\sum_{k} \frac{1}{m-1} g^{\prime i \bar{i}} g^{\prime j \bar{j}} \varphi_{i \bar{i} k} \varphi_{j \bar{j} \bar{k}} \\
& -\sum_{k} g^{\prime i \bar{i}} g^{\prime j \bar{j}} \varphi_{\bar{i} j k} \varphi_{i \bar{j} \bar{k}} .
\end{aligned}
$$

Using Cauchy-Bunyakowsky-Schwarz, we obtain

$$
\begin{aligned}
(m+\Delta \varphi)^{-1} g^{\prime i \bar{i}}(\Delta \varphi)_{i}(\Delta \varphi)_{i} & =(m+\Delta \varphi)^{-1} \sum_{i} g^{\prime i \bar{i}}\left(\sum_{k} \varphi_{k \bar{k} i}\right)\left(\sum_{k} \varphi_{k \bar{k} \bar{i}}\right) \\
& \leq(m+\Delta \varphi)^{-1} \sum_{i} g^{\prime i \bar{i}}\left(\sum_{k} \frac{\left|\varphi_{k \bar{k} i}\right|^{2}}{\left(1+\varphi_{k \bar{k}}\right)}\right)\left(\sum_{k}\left(1+\varphi_{k \bar{k}}\right)\right) \\
& =\sum_{i, k} g^{\prime i \bar{i}} g^{\prime k \bar{k}}\left|\varphi_{k \bar{k} i}\right|^{2} \\
& \leq \sum_{i, j, k} g^{\prime i \bar{i}} g^{\prime j \bar{j}}\left|\varphi_{i \bar{j} k}\right|^{2} .
\end{aligned}
$$

We are left with

$$
\begin{aligned}
f^{\frac{-1}{m-1}} \Delta\left(f^{\frac{1}{m-1}}\right) \leq & \alpha_{0} m(m+\Delta \varphi)+S-\left(\alpha_{0}+\inf _{i, k} R_{i \bar{i} k \bar{k}}\right)(m+\Delta \varphi)\left(\sum_{i} g^{\prime i \bar{i}}\right) \\
& +\sum_{k} \frac{1}{m-1} g^{\prime i \bar{i}} g^{\prime j \bar{j}} \varphi_{i \bar{i} k} \varphi_{j \bar{j} \bar{k}} .
\end{aligned}
$$

It is at this point that we use the hypotheses that $m=2$ and $f^{1 / 2}$ is Lipschitz continuous. From taking the derivative of both sides of $\left(\operatorname{det} g_{i \bar{j}}^{\prime}\right)^{1 / m}=\left(f \operatorname{det} g_{i \bar{j}}\right)^{1 / m}$, we see that $g^{\prime i \bar{j}} \varphi_{i \bar{j} k}=2 f^{-1 / 2} \partial_{k} f^{1 / 2}$. Therefore,

$$
f^{-1} \Delta f \leq 2 \alpha_{0}(2+\Delta \varphi)+S-\left(\alpha_{0}+\inf _{i, k} R_{i \bar{i} k \bar{k}}\right)(2+\Delta \varphi)\left(\sum_{i} g^{\prime i \bar{i}}\right)+4 \frac{\left|\nabla f^{1 / 2}\right|^{2}}{f} .
$$


Since we choose $\alpha_{0}$ such that $\alpha_{0}+\inf _{i, k} R_{i \bar{i} k \bar{k}}>0$, we use (3.14) and get

$-A \leq S \sup _{M} f+4\left\|\nabla f^{1 / 2}\right\|_{\infty}^{2}+2 \alpha_{0}\left(\sup _{M} f\right)(2+\Delta \varphi)-\left(\alpha_{0}+\inf _{i, k} R_{i \bar{i} k \bar{k}}\right)(2+\Delta \varphi)^{2}$.

As shown in the previous argument, it follows that $(m+\Delta \varphi) \leq C$.

\section{Dirichlet problem in $\mathbb{C}^{m}$}

As an application of the a priori estimates shown previously, we shall solve a Dirichlet problem in $\mathbb{C}^{m}$, following the footsteps of Caffarelli, Kohn, Nirenberg and Spruck [6]. In order to prove Theorem 3, we will make use of estimates previously established in the literature and combine them with our result.

Let $\Omega$ be a strongly pseudo-convex domain and $u$ be a strictly pluri-subharmonic solution to (1.3), where $f>0$. We let $\psi$ be a strictly pluri-subharmonic function on $\Omega$ such that $\psi=0$ on $\partial \Omega$ and

$$
\operatorname{det}\left(\psi_{i \bar{j}}\right)>\sup _{\bar{\Omega}} f \geq \operatorname{det}\left(u_{i \bar{j}}\right) .
$$

By a maximum principle such as the one given in [6], we have have $\psi \leq u$ in $\bar{\Omega}$. To get a upper bound, we solve the Laplace equation for a harmonic function $h: \Delta h=0$ in $\Omega$ and $h=0$ on $\partial \Omega$. Then since $\Delta u \geq 0$ in $\Omega$, we have $u \leq h$. Using $\psi \leq u \leq h$ in $\Omega$ and $\psi=u=h$ on $\partial \Omega$, we can obtain $|\nabla u(z)| \leq \max \{|\nabla \psi(z)|,|\nabla h(z)|\}$ for all $z \in \partial \Omega$. Since $\psi$ and $h$ depend only on $\Omega$, we have a gradient estimate on the boundary.

To push the interior gradient estimate to the boundary as done in [6], we need to introduce the additional hypothesis that $f^{1 / m}$ is Lipschitz. It is an open question to determine whether this assumption can be improved to requiring that $f^{1 /(m-1)}$ is Lipschitz. Such a result would lead to a more natural statement for Theorem 3, which would be analogous to the result given in [11] for the real Monge-Ampère equation. In our case, we assume the hypothesis that $\left|\nabla f^{1 / m}\right| \leq A_{1}$, and thus have

$$
\|u\|_{C^{1}(\bar{\Omega})} \leq C \text {. }
$$

We can obtain second-order estimates of $u$ from our current result. Define $\varphi:=$ $u-|z|^{2}$. We have $\operatorname{det}\left(\delta_{i \bar{j}}+\varphi_{i \bar{j}}\right)=f \operatorname{det} \delta_{i \bar{j}}$. Consider the test function $H=(m+$ $\Delta \varphi) e^{-\alpha(\varphi)}$ from Proposition 4. If $H$ attains its maximum on $\partial \Omega$, then $|m+\Delta \varphi| \leq$ $C\|m+\Delta \varphi\|_{L^{\infty}(\partial \Omega)}$. If $H$ attains its maximum at $p \in \Omega$, then we obtain $|m+\Delta \varphi| \leq C$ if we follow the proof of Proposition 4 with $g_{i \bar{j}}=\delta_{i \bar{j}}$. Therefore, we have

$$
\|\Delta u\|_{L^{\infty}(\bar{\Omega})} \leq C\left(1+\|\Delta u\|_{L^{\infty}(\partial \Omega)}\right) .
$$

Control of second-order derivatives on $\partial \Omega$ follows from the argument in [6], and this argument relies on a $C^{1}$ estimate and $f^{1 / m}$ to be Lipschitz. Therefore, we have the following result.

Proposition 6. Let $\Omega$ be a strongly pseudo-convex domain in $\mathbb{C}^{m}$. Let $f: \Omega \rightarrow \mathbb{R}$ be a function such that $f>0,\left|\nabla f^{1 / m}\right| \leq A_{1}$, and $\Delta f^{\frac{1}{m-1}} \geq-A_{2}$. Suppose there exists a strictly pluri-subharmonic solution $u \in C^{\infty}(\bar{\Omega})$ such that

$$
\begin{gathered}
\operatorname{det} u_{i \bar{j}}(z)=f(z) \text { in } \Omega, \\
u=0 \text { on } \partial \Omega .
\end{gathered}
$$


Then there exists a constant $C$ which depends only on $\Omega, \sup (f), A_{1}$ and $A_{2}$ such that

$$
\|u\|_{C^{1}(\bar{\Omega})}+\|\Delta u\|_{L^{\infty}(\bar{\Omega})} \leq C
$$

Using Proposition 6, we shall now prove Theorem 3. The strategy will be to solve the non-degenerate Dirichlet problem for $f>0$, and then use a limiting process. Let $g_{\varepsilon}=f^{\frac{1}{m-1}}+\varepsilon$, with $\varepsilon>0$. We extend $f$ such that it is defined on all of $\mathbb{C}^{m}$. Let $\gamma_{\rho}=\gamma(|z| / \rho)$, where $\gamma: \mathbb{C}^{m} \rightarrow \mathbb{R}$ is a $C^{\infty}$ function of compact support such that $0 \leq \gamma \leq 1, \int_{\mathbb{C}^{m}} \gamma=1$. We define $h_{\varepsilon, \rho}: \Omega \rightarrow \mathbb{R}$ in the following way:

$$
h_{\varepsilon, \rho}(x)=\left(g_{\varepsilon} * \gamma_{\rho}(x)\right)^{m-1} .
$$

Since $\bar{\Omega}$ is compact, we know that $g_{\varepsilon} * \gamma_{\rho} \rightarrow g_{\varepsilon}$ uniformly on $\bar{\Omega}$. For $\rho, \varepsilon>0$ small enough, it can be shown that

$$
\left|\nabla h_{\varepsilon, \rho}^{1 / m}\right| \leq 2 A_{1}, \quad \Delta h_{\varepsilon, \rho}^{1 /(m-1)} \geq-A_{2} .
$$

Now, we consider the non-degenerate Monge-Ampère Dirichlet problem

$$
\begin{gathered}
\operatorname{det}\left(u_{\varepsilon, \rho}\right)_{i \bar{j}}=h_{\varepsilon, \rho} \text { in } \Omega, \\
u_{\varepsilon, \rho}=0 \text { on } \partial \Omega .
\end{gathered}
$$

Caffarelli, Kohn, Nirenberg and Spruck [6], since $h_{\varepsilon, \rho}$ is smooth, we know that there exists a smooth strictly pluri-subharmonic solution $u_{\varepsilon, \rho}$. By Proposition 6, we have

$$
\left\|u_{\varepsilon, \rho}\right\|_{C^{1}(\bar{\Omega})}+\left\|\Delta u_{\varepsilon, \rho}\right\|_{\infty} \leq C
$$

for some constant $C$ independent of $\varepsilon$ and $\rho$. We let $\rho \rightarrow 0$ and obtain a strictly pluri-subharmonic solution $u_{\varepsilon}$ of

$$
\begin{aligned}
& \operatorname{det}\left(u_{\varepsilon}\right)_{i \bar{j}}=\left(f^{\frac{1}{m-1}}+\varepsilon\right)^{m-1} \text { in } \Omega, \\
& u_{\varepsilon}=0 \text { on } \partial \Omega .
\end{aligned}
$$

such that $\left\|u_{\varepsilon}\right\|_{C^{1}(\bar{\Omega})}+\left\|\Delta u_{\varepsilon}\right\|_{\infty} \leq C$. Finally, we let $\varepsilon \rightarrow 0$ and obtain a plurisubharmonic solution $u$ of (3) such that $\|u\|_{C^{1}(\bar{\Omega})}+\|\Delta u\|_{\infty} \leq C$. Uniqueness is well-known and can be found in [6].

\section{Acknowledgments}

The author would like to thank Professor Pengfei Guan for suggesting this problem, and for his invaluable advice and guidance. The author would also like to thank Xiangwen Zhang for pointing out several typos in the draft of this paper.

\section{References}

[1] T. Aubin, Equations du type Monge-Ampere sur les varietes kahleriennes compactes, C. R. Acad. Sci. Paris 283 (1976), 119-121.

[2] E. Bedford and B. Taylor, The Dirichlet problem for a complex Monge-Ampere equation, Invent. Math. 37 (1976), 1-44.

[3] Z. Blocki, Regularity of the degenerate Monge-Ampere equation on compact Kahler manifolds, Math. Z. 244 (2003), 153-161.

[4] Z. Blocki, A gradient estimate in the Calabi-Yau theorem, Math. Ann. 334 (2009), 317-327.

[5] S. Boucksom, P. Esyssidieux, V. Guedj and A. Zeriahi, Monge-Ampere equations in big cohomology classes, Acta Math. 205 (2010), 199-262. 
[6] L. Caffarelli, J. Kohn, L. Nirenberg and J. Spruck, The Dirichlet problem for nonlinear secondorder elliptic equations. ii. Complex Monge-Ampere, and uniformly elliptic equations, Commun. Pure Appl. Math. XXXVIII (1985), 209-252.

[7] X. Chen, The space of Kahler metrics, J. Differ. Geom. 56 (2000), 189-234.

[8] S. Donaldson, Symmetric spaces, Kahler geometry, and Hamiltonian dynamics, Northern California Symplectic Geometry Seminar, 196 (1999), 13-33.

[9] P. Esyssidieux, V. Guedj and A. Zeriahi, Singular Kahler-Einstein metrics, J. Amer. Math. Soc. 22 (2009), 607-639.

[10] H. Gu and Z. Zhang, An extension of Mok's theorem on the generalized Frankel conjecture, Sci. China Math. 53(5) (2010), 1253-1264.

[11] P. Guan, $c^{2}$ a priori estimate for degenerate Monge-Ampere equations, Duke Math. J. 86 (1997), 323-346.

[12] P. Guan, A gradient estimate for complex Monge-Ampere equation, Preprint, 2008.

[13] Z. Hou, Complex Hessian equation on Kahler manifold, Int. Math. Res. Not. 16 (2009), 3098-3111.

[14] S. Kolodziej. The Monge-Ampere equation on compact Kahler manifolds, Indiana Univ. Math. $\mathbf{5 2}(3)$ (2003), 667-686.

[15] D. Phong, J. Song and J. Sturm, Complex Monge-Ampere equations, Surv. Differ. Geom. 17 (2012), 327-411.

[16] D. Phong and J. Sturm, The Monge-Ampere operator and geodesics in the space of Kahler potentials, Invent. Math. 166(1) (2006), 125-149.

[17] Y. Siu, Lectures on Hermitian-Einstein metrics for stable bundles and Kahler-Einstein metrics, Birkhauser Verlag, Basel, 1987.

[18] S. Yau, On the Ricci curvature of a compact Kahler manifold and the complex Monge-Ampere equation, Commun. Pure Appl. Math. XXXI (1978), 339-411.

Department of Mathematics, McGill University, Montreal, Quebec H3A 2K6, Canada E-mail address: sebastien.picard@math.mcgill.ca 